\title{
A Bidirectional Scattering Function Reconstruction Method Based on Optimization of Microrelief Heights Distribution*
}

\author{
Vadim Sokolov ${ }^{1,2[0000-0002-1719-5102]}$, Dmitry Zhdanov ${ }^{1[0000-0001-7346-8155],}$ \\ Igor Potemin 1[0000-0002-5785-7465], Andrey Zhdanov ${ }^{1[0000-0002-2569-1982], ~}$ \\ Nikolay Deryabin 2[0000-0003-1248-6047] \\ ${ }^{1}$ ITMO University, 49 Kronverksky Pr., St. Petersburg, 197101, Russia \\ sokolovv1969@gmail.com, ddzhdanov@mail.ru, ipotemin@yandex.ru, \\ adzhdanov@corp.ifmo.ru \\ ${ }^{2}$ Keldysh Institute of Applied Mathematics RAS, Moscow, Russia \\ dek@keldysh.ru
}

\begin{abstract}
The work is devoted to the development of a new method for reconstructing the scattering properties of a rough surface, which is described using the bidirectional scattering distribution function (BSDF). There are several different methods of BSDF reconstruction using various approaches. However, they all have their drawbacks: for example, a method based on modeling the measured distribution of heights often requires a complicated fit apart from the expensive measurements themselves, various analytical methods are usually operable within the average roughness values with their standard distribution, and a rather good and universal method for optimizing the normals distribution density does not support internal reflections on the elements of the roughest surface. The proposed solution uses the geometry models of the rough surface, which allows simulating a physically more accurate propagation of light through the rough surface taking into account internal reflections, and hence a more accurate reconstruction of the bidirectional scattering distribution function. The results of BSDF reconstruction with the new method are proved by comparison with measurement results.
\end{abstract}

Keywords: Microrelief, Bi-directional Scattering Distribution Function, BSDF, Rough Surface, Diffusivity, Rendering, LGP, TIR, Wave Optics, Ray Optics

Copyright (C) 2020 for this paper by its authors. Use permitted under Creative Commons License Attribution 4.0 International (CC BY 4.0).

This research has been supported by the RFBR grants No. 1701-00363, 20-01-00547, Financial support of the leading universities of Russian Federation (subsidy 074-U01). 


\section{Introduction}

The optical elements with the rough surfaces are widely used in the modern devices with different aims, for example as sources to modify goniometric diagrams of light scattering, to obtain desired spatial luminance distributions for various lightguiding devices like luminaires with LED sources, car dashboards, illumination systems of displays, etc.

Also, the precise presentation of optical properties of rough surfaces is an important condition to obtain photorealistic images with different rendering tools for objects having such properties, and any simplification or ignoring important physical effects in the description of scattering properties can result in noticeable artifacts in generated images.

Besides the simplified methods of simulation of such objects as one-sheet layers with measured bi-directional scattering distribution function in many cases are not applicable because the thickness of elements with a rough surface cannot be ignored. So, the scattering properties of a rough surface must be extracted from both sides of the rough air-dielectric boundary. The measurements from both sides of the rough surface are hardly possible because of evident technical and optical problems. They are shown in Fig. 1. The first difficulty is to place a light detector inside of the material, to measure light distribution inside of the material. Another difficulty is to illuminate the rough surface from the material side. One more problem is to exclude parasitic light interreflections between the rough surface and another face of the measured sample.

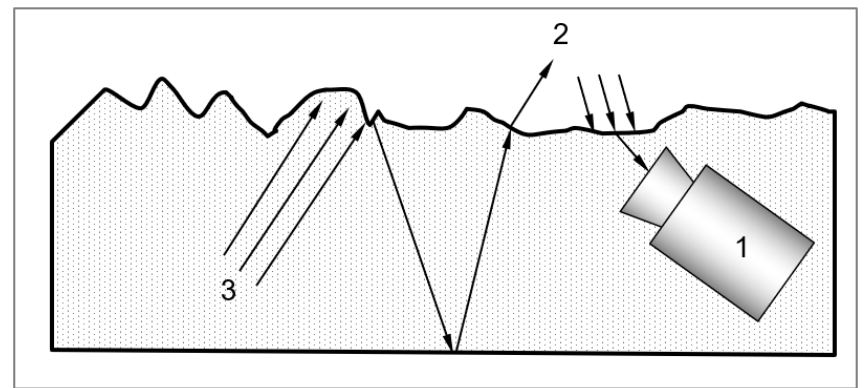

Fig. 1. The difficulties of BSDF measurement on a rough surface: (1) - a light detector inside of material; (2) - an illumination inside of material; (3) - a light interreflections inside of material.

All mentioned problems result in developing in a lot of approaches to reconstruct BSDF of the rough surface. Most of the existing methods to obtain scattering properties of the rough surface [1-9] are devoted to physically accurate reconstruction in comparison with MERL BRDF data. However physical accurateness of this database is not proved with information about certified equipment. Another drawback of developed methods is that substantial parts of these investigations are restricted with the BRDF component only while general BSDF including transmission component (BTDF) is required. A set of analytical approaches works well only for the average values of roughness while fails especially in the keeping of correct integral character- 
istics of general reflectance and transmittance for extremal values of small and big roughness. All these mentioned problems were a reason in the development of a new approach of BSDF reconstruction based on the optimization of normals density distributions. The approach is described in [10] and gives good results for a wide set of samples with different roughness however all estimations are done based on comparison with measured transmittance and reflectance for different illumination angles only. There is no real proof what the developed BSDF representation is sufficient for all possible applications up to the generation of photorealistic images.

Investigations of the given paper are extended with the generation of photorealistic images which allows us to do more reasonable conclusions about the drawbacks of the "Normals" approach. Theoretically, there is a drawback in the reconstruction method based on normal distribution density. The light propagation for this approach does not consider the spatial shape of the rough surface. In other words, only an angular light transformation is simulated according to the angular distribution of normals density. It results in ignoring such effects as light interactions with different facets of rough surface profile, see fig. 2

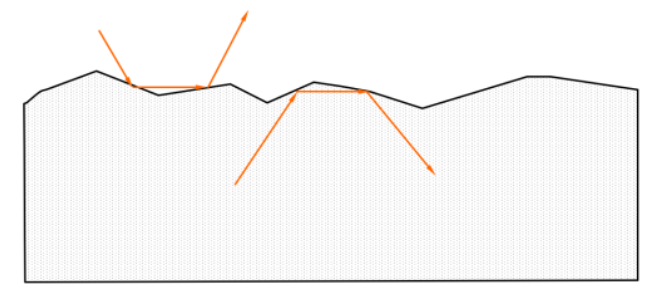

Fig. 2. Interreflections on profile of rough surface

Such approximation can result in overestimation or underestimation of reflected/transmitted light scattering specially for samples with substantial roughness and grazing illumination angles.

To check the "Normals" method presented in the article [10] the sample with average microroughness has been selected. Its BSDF was measured with GCSM-4 goniospectrophotometer [11] and reconstructed with help of "Normals" method. Then these BSDF were used to calculated transmitted intensity. In the case of measured BSDF the one sheet model was used like measurements of GCSM-4 (with ignoring sample thickness). The reconstructed BSDF was specified on a solid model of sample (attached to one of faces of a plate). The parameters of detector were the same for both cases. The fig. 3. presents transmitted light intensity for both BSDF: measured with GCSM-4 and simulated with "Normals" method [10].

Fig. 3 presents angular intensity for six light incident directions: $0^{\circ}, 15^{\circ}, 30^{\circ}, 45^{\circ}$, $60^{\circ}$, and $75^{\circ}$ (angles between normal to sample surface and incident light direction). The light illumination was executed from a smooth (polished) sample size, so the intensity was measured from the rough side. According to Fig.3, the agreement between transmitted intensity for reconstructed and measured BSDF is very good especially for incident light directions close to normal. Note there are some difference incident light directions far from normal, but it can be explained with more degree 
with measurements errors than an approximation of BSDF reconstruction because errors of GCSM-4 measurements can be significant for grazing light directions because of the finite spot of light illumination, observation and light leakage along sample surface.

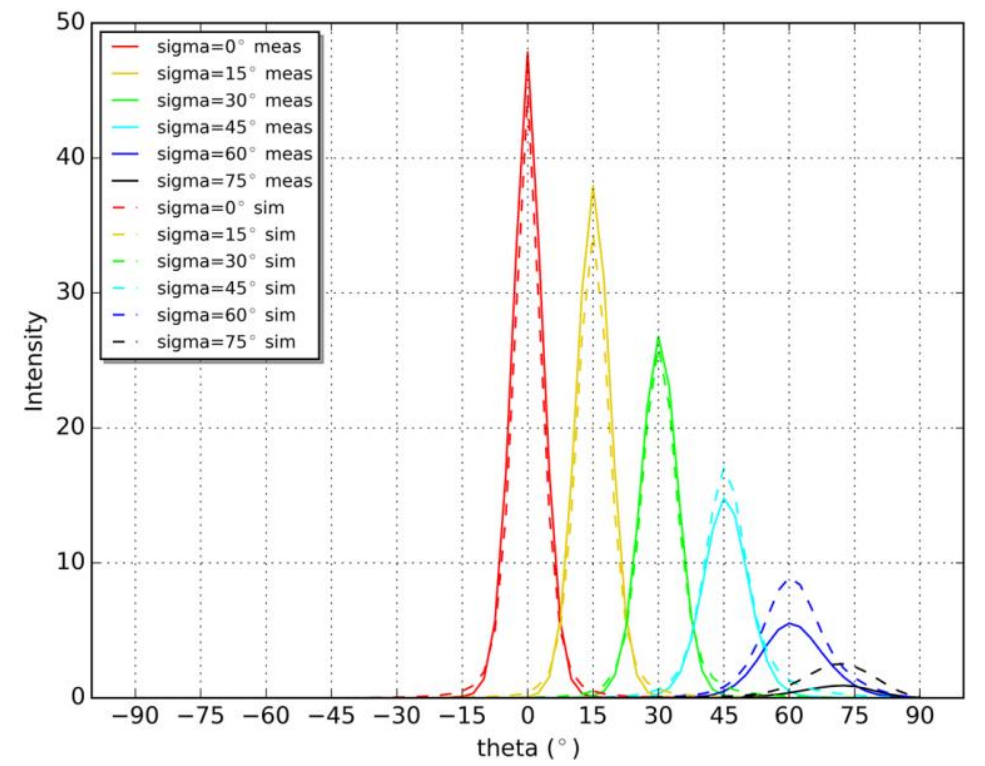

Fig. 3. Transmitted light intensity for sample measured with GCSM-4 vs. simulated with BSDF reconstructed with 'Normals' method

The next fig.4 presents an image of some test objects with specified BSDF simulated with rendering. Three test objects: a plate, a sphere, and an object with a more complex shape -a teapot has a rough external surface. The thickness of the walls is small. It is done intentionally to have the possibility to verify the object's appearance with measured BSDF (solid modeling can be replaced with one-sheet). The objects are placed in a diffuse white box with illumination close to diffuse (several tubeshaped light sources with self-emitting are placed above the box ceiling). For better appearance, the test objects are placed on substratum with chessboard like texture

The image presented in fig. 4 was generated with Path Tracing rendering as a more physically accurate and fast tool available in Lumicept $[12,13]$ which uses a hybrid raytracing technique with mutual usage of the forward Monte-Carlo and backward deterministic ray tracing. The tool allows generating images of photorealistic quality for scenes with objects having complex properties and complex illumination. The image with measured BSDF is very similar and not presented in the paper, however, some effects like bright contours on the teapot and sphere (marked with red arrows on zoomed fragments) are absent on it. The most likely reason for these artifacts is the ignoring of interreflection effects with the "Normals" method which were explained above. Thus, simulated images with reconstructed BSDF show the noticeable defects 
on some samples with significant roughness and guide to improvements. The topic of the paper is a presentation of a new improved method for BSDF reconstruction.

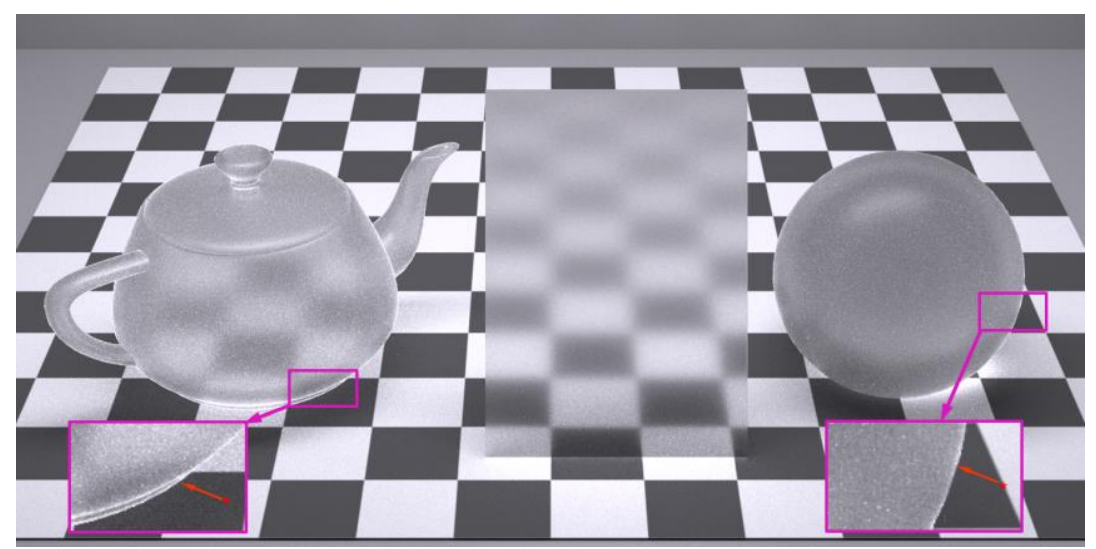

Fig. 4. An image of several test objects for BSDF reconstructed with the "Normals" density distribution method simulated with rendering

\section{The new method of BSDF reconstruction based on micro- profile geometry simulation}

There are two base methods of numerical BSDF reconstruction. The method based on measurements or heights distribution of rough surface profile and BSDF of the whole sample (transparency or/and reflectance) [14]. This approach does not give guaranteed good output and requires complex optimization of the micro profile (reducing to scaling or filtration of the profile). As an alternative to the method requesting expensive measurements of heights distribution an approach with optimization of normal density distribution was introduced in [10] The last approach is cheaper and flexible however, does not support some effects like interreflections on microprofile faces shown in the fig. 2 .

The main idea of the new approach is to combine benefits of both numerical approaches explained above: to exclude measurements of profile distribution and support maximally all physical effects of light transformation through profile during the reconstruction procedure. "Normals" method uses an analytical function to describe normal density distribution and use it in BSDF reconstruction. Why do not use a similar approach in the specification of height distribution with similar analytical functions? It is illustrated with fig. 5. It shows a regular grid of points with uniform pitches along $\mathrm{x}$ and $\mathrm{y}$ axes. Each point in the grid presents a node of micro-profile. To define profile height in each node with $\mathrm{x}$, $\mathrm{y}$ coordinates the analytical probability function of one or several parameters that can be used

$$
H(x, y)=\operatorname{Prob}(F)
$$


6 V. Sokolov, D. Zhdanov, I. Potemin, et al.

In other words, height in each node is defined according to some probability defined according to normal (Gauss) or some other analytical function specifying height density distribution. In the paper the same two functions were used: "Gauss" like, see formula (2), and "Cauchy" like, see formula (3

$$
\begin{gathered}
F_{\text {gauss }}=H_{\text {max }} e^{\left(-\frac{\left(z-z_{0}\right)^{n}}{2 \sigma^{n}}\right)} \\
F_{\text {cauchy }}=H_{\max } \frac{\sigma^{n}}{\left(z-z_{0}\right)^{n}+\sigma^{n}}
\end{gathered}
$$

Both functions depend on four parameters $\left(\sigma, H_{\max }, n\right.$, and $\left.\mathrm{z}_{0}\right)$. It is a rather substantial parameter number, which can complicate the process of optimization convergence however experiments show most of the cases the only $\sigma$ (sigma) is sufficient, " $n$ " (degree) can slightly improve convergence in some cases. $H_{\max }$ can be set as 1 most of the cases if to set pitch between nodes of the profile grid around the same unit value. $\mathrm{z}_{0}$ is supposed to be zero (density of heights is symmetrical relative to $H_{\max }$ ). $\mathrm{z}$ is in range $0-H_{\max }$. So $F$ defines the distribution of height density.

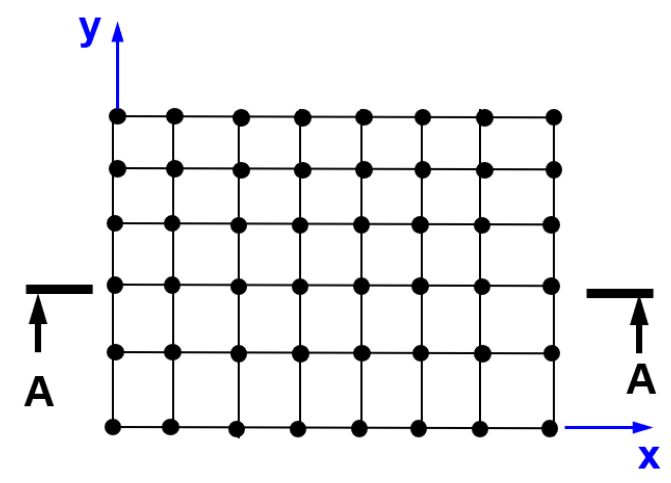

A-A section

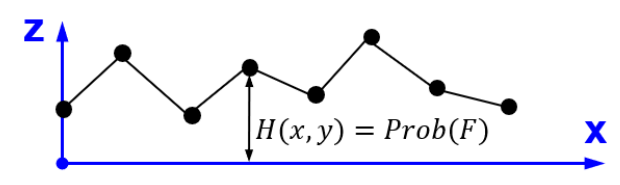

Fig. 5. Definition of heights distribution with analytical functions

According to formula (2) or (3) height distribution of micro profile can be defined and used for profile geometry generation, see fig. 6. 

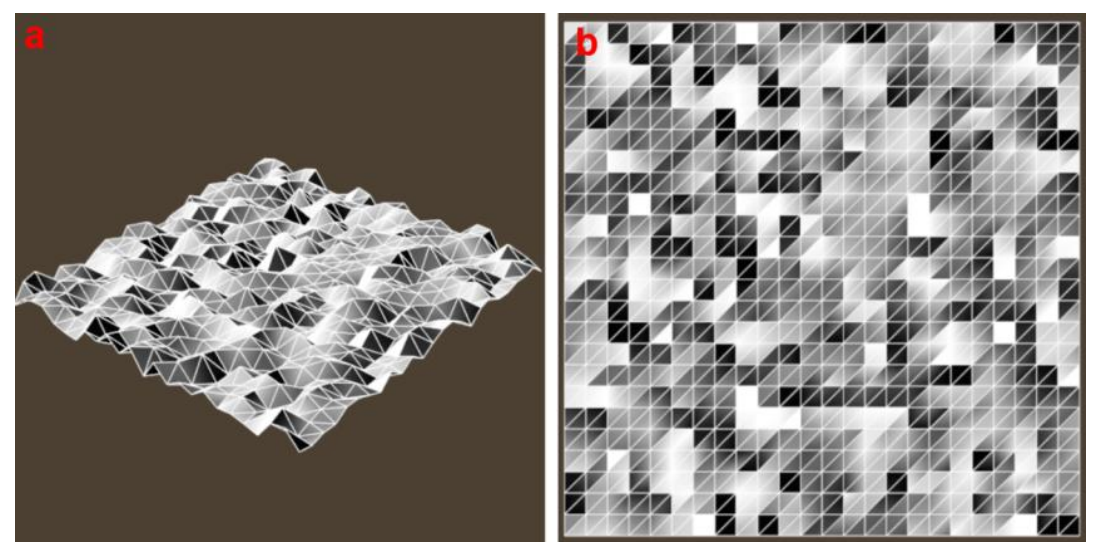

Fig. 6 A schematic appearance of micro profile based on analytical heights distribution: (a) - perspective view; (b) - top view

The new approach (named here as 'Height' method) is based on the only kind of data: BSDF measured for the entire sample. Note measured transparency or/and reflectance of a plane sample measured as a one-sheet element. Despite the difference with the 'Normal' algorithm base model of the new approach is the same as described in [10]. The source of the reconstructed BSDF is an intensity distribution calculated after the ray transformations on the microfacets boundary of two media. The only difference is that microfacets are defined as a height distribution. Application of the OPTOS MicroHeight tool [15] of Lumicept [12] provides physically accurate calculations of the intensity distribution, scattered on the microrelief.

It should be pointed out what BSDF defined in whole $3 \mathrm{D}$ space is a very complex function with a lot of degrees of freedom. Its calculation is a rather long process. To accelerate the process of optimization of height distribution (searching of optimal parameters to find desired height distribution) it is used a simplification. For BSDF reconstruction a real flat sample (plate) is used in which one of the surfaces is smooth and the other is rough. The plate is illuminated by a collimated beam of light. Usually, one or two incident directions are used (sigma $=0,30$ as an example). The scattered light is calculated also for each incident light direction in a single plane only (the plane of light incidence). Standard gonio-diagram detectors of the Lumicept program complex [12] are used in the calculation. To agree measured data with reconstructed ones an additional model is simulated. A one-sheet plane with measured BSDF is calculated with the same illumination and observation conditions what and solid model used for reconstruction the same illumination and observation conditions are used. Such an approach provides a trivial way to obtain objective function which can be used on the process of optimization of profile height distribution. 


\section{Optimization of BSDF reconstruction procedure based on the 'Heights' distribution}

Like in the 'Normal' method an initial specification of parameters based on the measured BSDF cannot provide a good solution. An optimization process is required and very similar to optimization with the 'Normal' method [10]. The optimization scheme is illustrated with fig. 7.

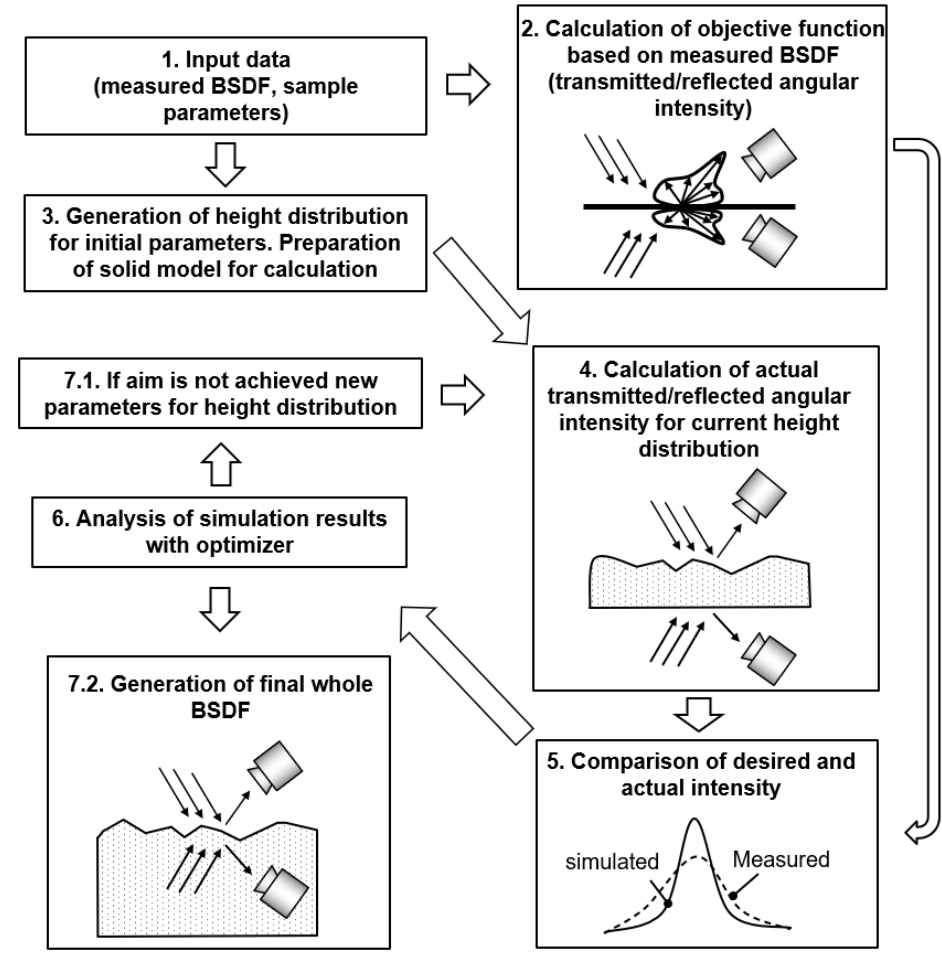

Fig. 7 Optimization scheme of BSDF reconstruction

In other words, height in each node is defined according to some probability defined according to normal (Gauss) or some other analytical function specifying height density distribution.

The optimization procedure consists of the following step:

1. The first step is an input of all required data: measured BSDF of the whole sample, sample sizes, refractive index, and transparency of the sample medium. 
2. The objective function is calculated in the second step. Measured BSDF is attached to a one-sheet surface, then it is illuminated with parallel light under one or several light incident angles and the Intensity of transmitted and/or reflected light is calculated in the plane of light incidence. These data are used as "etalon" to compare with calculated ones during the optimization process

3. During the third step, the solid model of the sample is creates based on input data. a profile is formed according to the height distribution function with initial parameters and specified on one of the sample sizes with OPTOS MicroRelief plugin [15].

4. Then intensity distribution is calculated under illumination and observation conditions identical to etalon data.

5. Further, measured and simulated results are compared, and RMSD (Root-Mean Square Deviation) between them is calculated.

6. RMSD (as final optimization criterion) and parameters of the height distribution function is transferred to the optimizer. It analyses obtained data and based on RMSD value:

7.

7.1. if the optimizer does not reach the desired deviation, then the optimizer changes parameters of the distribution density of normals and goes to step to continue the process.

7.2. Afterwards, if deviations are suitable, the final BSDF is generated with the help of "BSDFCalculator" integrated into the Lumicept [12] tool.

Note several open-source optimizers from SKIPY/NUMPY libraries were tested during this optimization. More good convergence was obtained with the LevenbergMarquardt method [16]. It requires a rather small number of steps (10-20 mostly) to achieve the desired output.

\section{Comparison of results of BSDF reconstruction both 'Normals' and 'Heights' methods}

The results of BSDF reconstruction with the "Normals" method have been presented in fig. 3 (intensity of transmitted light) and fig. 4 (render image). Fig, 8 presents the angular intensity distribution of transmitted light for the 'Heights' method. 
10 V. Sokolov, D. Zhdanov, I. Potemin, et al.

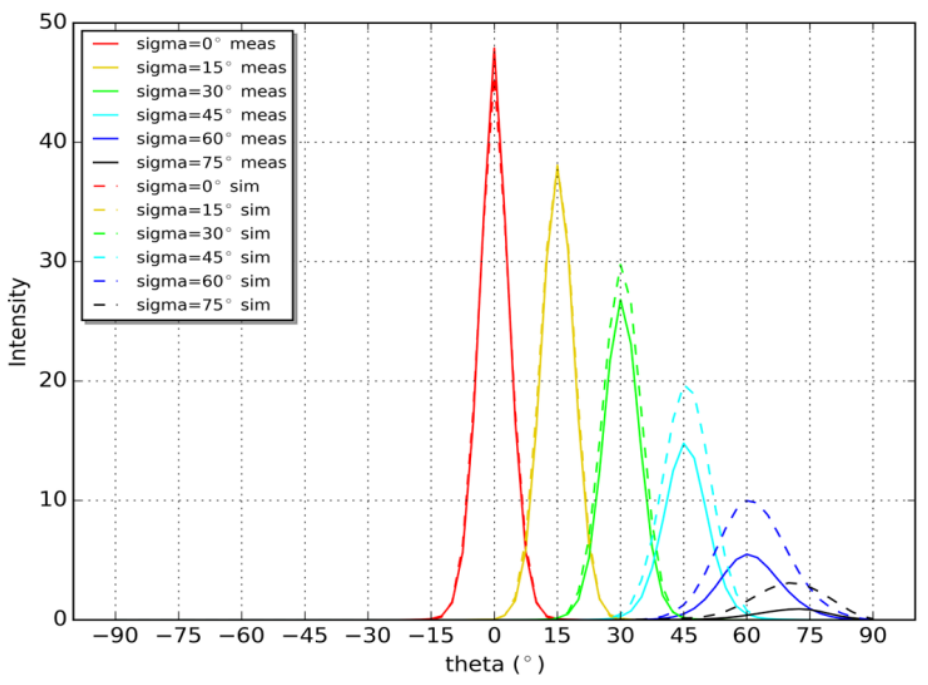

Fig. 8 Transmitted intensity for sample measured with GCSM-4 vs. simulated with BSDF reconstructed with 'Heights' method

All conditions to obtain the angular intensity of transmitted light presented in fig. 8 to evaluate the "Height" method of BSDF reconstruction corresponds to similar graphs presented in fig. 3 for the "Normals" approach. Note also simulated intensity for the new 'Height' method is rather like intensity obtained for measured BSDF and it is close to the" Normals" approach. Fig. 9 presents a render image of test objects with BSDF reconstructed with the 'Heights' method.

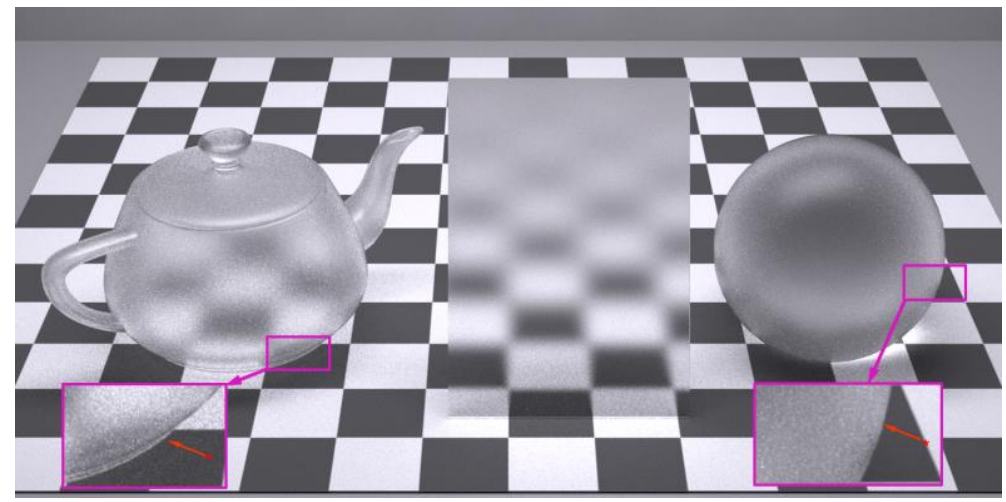

Fig. 9 An image of several test objects generated for BSDF reconstructed with the "Heights" distribution method

A comparison of images in fig 4 and 9 show some difference in appearance of test objects. It is not very considerable strong but rather noticeable. The essence is the 
absence of bright contour around the test object in fig. 9. Our investigations showed what a source of "bright-contour" artifact is the BRDF component from the material side. The shape of this BRDF component for "Normals" vs. "Heights" is presented in fig. 10 .

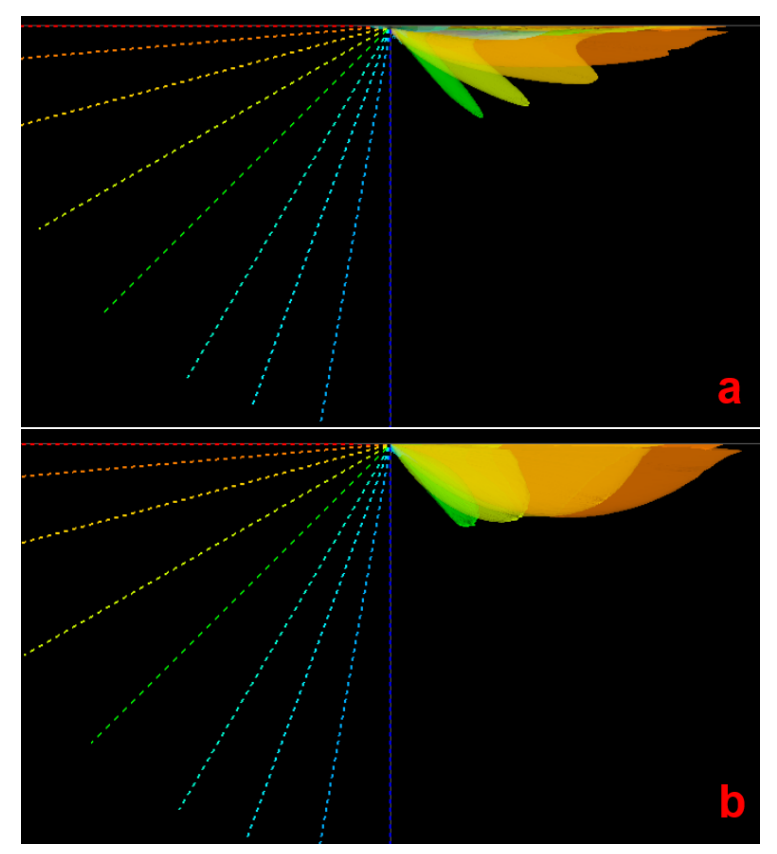

Fig. 10 BRDF plots. (a) for the "Normals" approach, (b) for the "Heights" approach

Fig. 10 presents BRDF plots (in solid colors) for the several incident light directions (marked with the same color of dash lines). BRDF in fig.10 presents the distribution of luminance factor reduced in logarithmical scale for better visualization. It is noticeable what BSDF of "Normals" and "Heights" approached are different in shape. In any case, as it was mentioned the artifacts are absent on images with measured BSDF. Thus, it shows the advantage of the "Heights" method vs. "Normals". The Cauchy like function (2) to describe the probability of heights distribution was used for the reconstruction of the presented sample. The investigation shows the function wins respecting to Gauss distribution on most of the BSDF samples. The conclusion is in agreement with outputs done for the "Normals" reconstruction method done in [10].

\section{Features of the "Heights" method}

It is worth to mention some specificity of the "Heights" method of BSDF reconstruction. The main difference of the "Heights" method from "Normals" is a more 
realistic special representation of profile instead of simplified just angular normal density. But the spatial representation of the profile requires a more complicated procedure of simulation with a greater number of variables. Several questions appear here, for example as many points (grid nodes, see fig.5) are required to represent the profile and how to link these points into the geometry of the profile. Any light simulators work not just with points but with some presentation of geometry (triangular, NURBS, etc. presentations). "OPTOSHeights" plugin of Lumicept [12] used in the given paper triangular presentation. The simplest solution is to link nodes of a selected regular grid with triangles. But as shows investigations done in the scope of the work such solution (see fig11 a) has a serious drawback: profile geometry generated with such an approach has artificial regularity which results in substantial artifacts on images generated with such reconstructed BSDF. For example highlight zones on rough surfaces with curvature will have a rectangular shape instead expected circular one. A more natural idea to suppress these problems is the usage of interpolation, see fig. $11 \mathrm{~b}$.
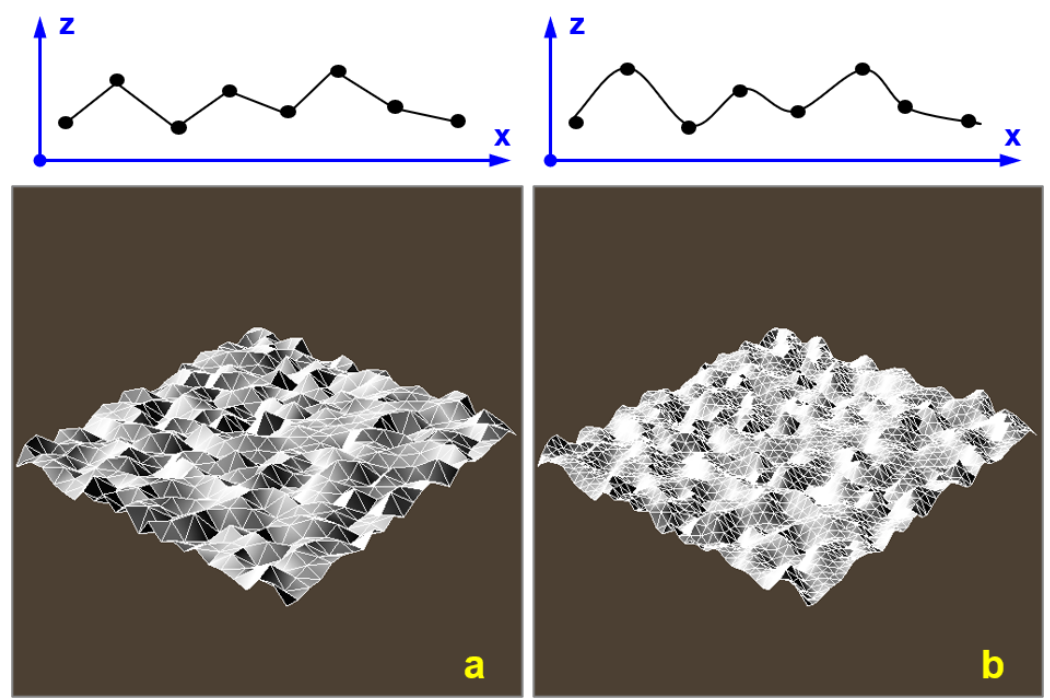

Fig. 11 A profile presentation without (a) and with (b) interpolation

The investigations show that it is sufficient to generate a profile with a grid with a resolution in range 500x500 - 1000x1000. The resolution of the mesh representing the profile should be increased in 2-4 times with linear or cubic interpolations between nodes. The next increase of resolution parameters does nor results in substantial-quality gain while too high-resolution results in slowing down the process of BSDF reconstruction. 


\section{Conclusion}

The results of BSDF reconstruction with the new "Heights" method show good agreement with measurements as on the quantitative level (angular intensity of scattered light) as well with the qualitative level (render images). Artifacts appeared in the application of the "Normals" method disappear on the images rendered with the proposed "Heights" method. Apart from the simulation of real profile geometry with the "Heights" method is more accurate from the viewpoint of light simulation as interreflections on profile micro facets are considering. The new method is more flexible to possible extensions relative to "Normals". The shape of rough surfaces can be more complex or artificial to describe it with analytical functions of normal density or heights density and generation of profile geometry is a more open tool to this challenge. Other methods can be invented for profile generation as an example with a random filling of spheres or objects repeating tool shape using for profile formation. As in the case of the "Normals" method, the profile measurements are not required and it is also a noticeable advantage of the approach. Note also there is some difference in simulated transparency vs. measured one on grazing incident angles and observed for both "Normals" and "Heights" approaches. The most likely reason is the inaccuracy of measurements with the GCSM4 device [11]. The measurements of the device for incident illumination angles greater than 45 degrees are less accurate. Another less likely reason is the absorption of transmitted light which may be more significant for big incident angles and it is ignored in simulation models. There can be a question of why some analytical functions with a restricted number of parameters (a degree of freedom) have been selected instead of some more general tabular functions with an unlimited number of parameters. The answer is evident for the sake of better convergence during the optimization process. The real ray propagation is used for BSDF calculation and it is not an instant process, so it is very preferable to restrict the optimization process with a restricted number of design steps (tens but not thousands or greater). Apart from the BSDF function has a rather complex shape and very sensitive. So, the selection of arbitrary tabular functions results in serious complications of optimization. On the other side, some correcting with tabular function as in the case of the "Normals" approach described in [10] is possible also. However, this narrow technical aspect was out of the paper and planed in the next investigations.

\section{References}

1. Nurcan Seylan, Serkan Ergun, Aydın Öztürk "BRDF Reconstruction Using Compressive Sensing", 21st International Conference on Computer Graphics, Visualization and Computer Vision - pp. 88-94. ISBN 978-80-86943-74-9 (2013)

2. Jannik Boll Nielsen, Henrik Wann Jensen, Ravi Ramamoorthi "On Optimal, Minimal BRDF Sampling for Reflectance Acquisition”, ACM TOG 34(6), pp. 1-11 (2015).

3. Doris Antensteiner and Svorad `Stolc "Full BRDF Reconstruction Using CNNs from Partial Photometric Stereo-Light Field Data", Workshop on Light Fields for Computer Vision at ECCV-2017, pp. 13-21 (2017). 
14 V. Sokolov, D. Zhdanov, I. Potemin, et al.

4. Feng Lu, Xiaowu Chen, Imari Sato, and Yoichi Sato "SymPS: BRDF Symmetry Guided Photometric Stereo for Shape and Light Source Estimation”, IEEE Transactions

5. Manmohan Chandraker, Jiamin Bai, and Ravi Ramamoorthi "On Differential Photometric Reconstruction for Unknown, Isotropic BRDFs", IEEE Transactions on Pattern Analysis and Machine Intelligence, Vol. 35, No. 12, pp. 2941-2954 on Pattern Analysis and Machine Intelligence, Issue: 99, pp. 1-14 (2013).

6. Sing Yee Chuaa, Xin Wanga*, Ningqun Guoa, Ching Seong Tan "Performance of Range Gated Reconstruction: A Theoretical Analysis", Proc. of SPIE, Vol. 10250, pp. 1-5.

7. Jirí Filip, Michal Havlí, Radomír Vávra "Adaptive highlights stencils for modeling of multi-axial BRDF anisotropy"// The Visual Computer, Volume 33, Issue 1, pp. 5-15 (2017)

8. Guojun Chen, Yue Dong, Pieter Peers, Jiawan Zhang, Xin Tong "Reflectance Scanning: Estimating Shading Frame and BRDF with Generalized Linear Light Sources", OOPSLA '94, Volume 5, Issue 4, October, pp. 67-71 (1994).

9. Wojciech Matusik, Hanspeter Pfister, Matt Brand and Leonard McMillan "A Data-Driven Reflectance model"// ACM Transactions on Graphics 22, 3, 759-769 (2003).

10. Bogdanov N., Zhdanov A.D., Zhdanov D.D., Potyomin I.S., Sokolov V.G., Denisov E.Y. A Bidirectional Scattering Function Reconstruction Method Based on Optimization of the Distribution of Microrelief Normals // Light \& Engineering, Volume 27, №1 (2019)

11. Muracami Color Research Laboratory, http://www.mcrl.co.jp/english/index.html, last accessed 2017/08/20

12. Lumicept - Hybrid Light Simulation Software, http://www.integra.jp/en, last accessed 2020/09/01

13. Zhdanov, D.D., Garbul, A.A., Maiorov, V.A., Potemin, I.S., Sokolov, V.G. Indeterminate ray tracing in problems of the analysis of light scattering and the design of illuminating systems, J. Opt. Technol. Vol. 81, Issue 6, pp. 322-326. DOI:10.1364/JOT.81.000322, (2014).

14. Sokolov, V.G., Zhdanov D.D., Potemin, I.S., Garbul, A.A., Voloboy, A.G., Galaktionov, V.A., Kirilov N.. Reconstruction of scattering properties of rough air-dielectric boundary, Optical Review. 23(5), 834-841, DOI: 10.1007/s10043-016-0250-6, (2016)

15. Ershov S.V., Zhdanov D.D. Efficient application of Optical Objects in light simulation software // Proc. 15th International Conference on Computer Graphics and Applications GraphiCon-2005, Russia, Novosibirsk, June 20-24, p. 284-288 (2005).

16. Marquardt, Donald. An Algorithm for Least-Squares Estimation of Nonlinear Parameters".SIAM Journal on Applied Mathematics, (1963) 